while 279 (36\%) had impaired glucose tolerance and 152 (20\%) had newly detected diabetes. The outcome of MACE are described in Abstract 147 table 1. The analysis shows a significantly higher incidence ( $p<0.05$, HR 1.56, CI 1.15 to 2.13) of adverse cardiovascular outcome in patients with impaired glucose tolerance as well as diabetes (post challenge hyperglycaemia).

Conclusion The study concludes that a significant proportion of patients who present with ACS have abnormal glucose tolerance. Patients with abnormal glucose tolerance have significantly higher incidence of adverse cardiovascular outcome.

Abstract 147 Table 1 Predictive variables associated with adverse cardiovascular events

\begin{tabular}{lrl}
\hline Variable & p Value & HR (95\% Cl) \\
\hline Age & $<0.001$ & $1.04(1.03$ to 1.06$)$ \\
Discharged on $\beta$-blocker & 0.003 & $0.64(0.47$ to 0.86$)$ \\
Abnormal glucose tolerance & 0.005 & $1.56(1.15$ to 2.13$)$ \\
Past history of MI & 0.013 & $1.87(1.14$ to 3.08$)$ \\
Hypertension & 0.031 & $1.37(1.03$ to 1.84$)$ \\
Discharged on ACE-l/ARB & 0.041 & $0.63(0.40$ to 0.97$)$ \\
Discharged on aspirin & 0.067 & $1.64(0.96$ to 2.80$)$ \\
Hypercholesterolaemia & 0.159 & $0.80(0.60$ to 1.08$)$ \\
Gender (male) & 0.217 & $1.22(0.89$ to 1.69$)$ \\
Smoking status (current smoker) & 0.410 & $1.13(0.83$ to 1.54$)$ \\
Revascularisation & 0.478 & $0.90(0.66$ to 1.22$)$ \\
\hline
\end{tabular}

Results of survival analysis using Cox regression with $p<0.5$.

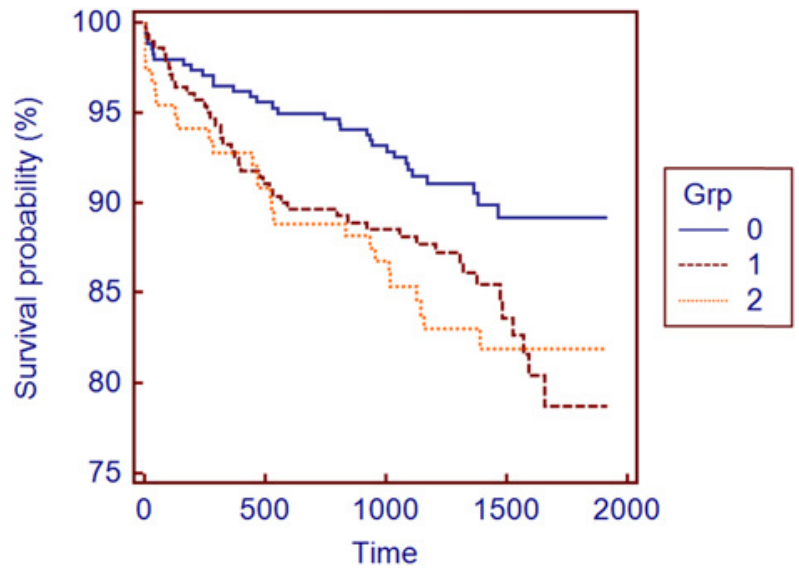

Abstract 147 Figure 1 Life status for MedCalc.

\section{INSULIN DEPENDENT DIABETES RESULTS IN WORSE OUTCOMES COMPARED TO NON-INSULIN DEPENDENT DIABETES FOLLOWING CORONARY ARTERY BYPASS GRAFT SURGERY (CABG)}

doi:10.1136/heartjnl-2012-301877b.148

K S Rathod, * S M Gallagher, S Hassan, M J Lovell, D A Jones, V S Rathod, D Bromage, R Uppal, C Knight, A Mathur, A Wragg, A Kapur. Barts and The London NHS Trust, London, UK

Aims Previous research has demonstrated that patients with diabetes mellitus (DM) have a worse prognosis than patients without diabetes following coronary artery bypass graft surgery (CABG). However, patients with insulin treated diabetes (ITD) may have a different prognosis when compared with non-insulin treated diabetic patients (NITD) after CABG. There is limited data investigating long term outcomes following CABG in patients with ITD and NITD patients. This study compared outcomes at 5 years following $\mathrm{CABG}$ in these two patient groups.

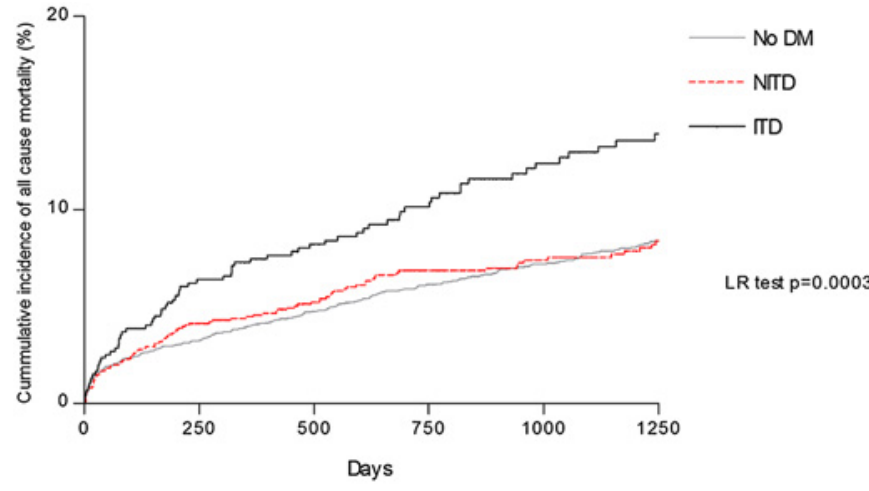

Numbers at risk

$\begin{array}{llllllr}\text { No DM } & 3924 & 3717 & 3238 & 2711 & 2294 & 1843 \\ \text { NITD } & 1257 & 1168 & 974 & 793 & 646 & 525 \\ \text { ITD } & 595 & 538 & 475 & 381 & 319 & 258\end{array}$

Abstract 148 Figure 1 All cause mortality after coronary artery bypass graft.

Methods 7442 consecutive patients underwent CABG at a single cardiac centre between 2003 and 2011. We identified 2471 patients with DM within this group. Demographic and procedural data were collected at the time of intervention. All cause mortality data were obtained from the Office of National Statistics via the BCIS/CCAD national audit out to a median of 2.80 years (CI 2.08 to 3.56 years). Results Of 2471 patients with DM, 741 (30.0\%) were ITD and 1730 $(70.0 \%)$ were NITD. ITD patients had significantly higher rates of previous MI $(65 \%$ vs $52 \%, \mathrm{p}<0.0001)$ and higher rates of renal disease $(10 \%$ vs $3 \%, p<0.0001)$. There were more female patients in the ITD group compared to the NITD patients (26\% vs $20 \%$, $\mathrm{p}=0.001)$. There was no difference in Age, rates of Hypertension, Hypercholestrolaemia, Previous PCI, or In-Hospital MACE between the two groups. At 5 years, there was no difference between nondiabetic patients and the NITD $(p=0.63)$. However, by 5 years all cause mortality was greater in the ITD group than in the NITD group $14 \%$ vs $9 \%(p<0.001)$. After adjusting for comorbidities, using multivariate analysis, ITD remained an independent predictor of long-term mortality (HR 1.64, 95\% CI 1.19 to 2.25 , p<0.002).

Conclusions Our data suggest that insulin treatment is an independent predictor of long term mortality for patients undergoing CABG. Treatment with insulin rather than diabetic status alone is an important factor affecting outcome in patients with coronary artery disease requiring surgical intervention. Furthermore, these differences in mortality appear after the first year. Hence there is a role for intensive medical therapy in ITD patients and further research is required to confirm these outcomes.

\section{ETHNIC DIFFERENCES IN PERFORMANCE OF THE 2010 EUROPEAN SOCIETY OF CARDIOLOGY CRITERIA FOR ECG INTERPRETATION IN ATHLETES}

doi:10.1136/heartjnl-2012-301877b.149

N Sheikh, ${ }^{*}$ M Papadakis, S Ghani, L Millar, R Bastiaenen, A Zaidi, S Gati, N Chandra, N Emmanuel, S Sharma. St. George's University of London, London, UK

Background Physical activity is associated with ECG phenotypes that may overlap with those observed in conditions predisposing to sudden cardiac death. In 2005 the study group of sports cardiology produced guidelines to differentiate ECG changes likely to reflect physiological adaptation to exercise from those, which should prompt further investigations. The guidelines were updated in 2010 resulting in improved specificity in predominantly Caucasian cohorts (white athletes; WA). We sought to examine the performance of the 2010 guidelines in athletes of African/Afro-Caribbean origin (black athletes; BA). 
Methods ECG of 923 male BA were evaluated to determine the proportion of individuals requiring further investigations based on the 2005 , compared to the updated 2010 guidelines. The same evaluation was performed for a cohort of 1711 male WA and 209 patients with hypertrophic cardiomyopathy (HCM). In addition, the impact of "Refined Criteria" was examined, consisting of an upper limit of 470 msec for QTc and removing the following as abnormalities: (1) isolated voltage criteria for left atrial enlargement (LAE); (2) Isolated voltage criteria for right ventricular hypertrophy (RVH); (3) T-wave inversions (TWI) in V1/2 in WA and V1-V4 in BA.

Results Using 2005 guidelines, 549 BA (59.5\%) met criteria for a positive ECG requiring referral for further investigations compared to 846 WA (49.4\%). In comparison, 398 BA (43.1\%) met criteria for a positive ECG using 2010 guidelines [predominantly due to TWI (22.3\%), RVH (13.0\%) and LAE $8.5 \%)$ ] compared to 216 WA $(12.6 \%)$ [predominantly due to TWI (3.0\%), RVH (2.8\%) and LAE $(2.7 \%)$ ]. All of the HCM patients met the criteria for a positive ECG, regardless of which guidelines were used. Using our "Refined Criteria," the number of BA with a positive ECG was reduced to 161 $(17.4 \%)$ and WA to 93 (5.4\%). Five patients with HCM had isolated voltage criteria for LAE (2.4\%); all were symptomatic apart from 1 $(0.5 \%)$. Five patients with HCM had voltage criteria for LAE in combination with LVH but no other abnormalities on their ECG; all were symptomatic apart from $1(0.5 \%)$.

Conclusions Updated guidelines significantly reduce the number of positive ECG results in WA, but less so in BA, emphasising the need for ethnicity specific criteria to be developed. Refining criteria based on physiological changes known to occur in athlete's heart results in further reduction in positive ECGs. Our findings in patients with HCM suggest that if found in isolation or in combination with voltage criteria for LVH alone, ECG evidence of LAE may be regarded as a physiological rather than pathological change.

\section{0 "HEART TEAM" DECISION MAKING IN THE MANAGEMENT OF PATIENTS WITH CORONARY ARTERY DISEASE; STRUCTURE, OUTCOMES AND REPRODUCIBILITY}

doi:10.1136/heartjnl-2012-301877b.150

L D Tapp,* J Thekkudan, E Morris, H Luckraz, M S Norell. New Cross Hospital, Birmingham, UK

Introduction Contemporary national and international guidelines recommend that patients with stable angina and acute coronary syndromes (ACS) with multi-vessel disease are discussed by a multidisciplinary "Heart Team" (HT) to facilitate optimal evidencebased management. However, there is a paucity of data describing the workings of a HT, actioning of it's recommendations and reproducibility of decisions.

Methods We have utilised a HT approach since 2005, meeting twice weekly with our HT database managed by a dedicated co-ordinator. We analysed the data for 2010 and describe the HT process. A random sample of cases were scrutinised to identify whether the HT decision had been implemented. Additionally, cases were represented to the HT after 1 year to determine consistency and reproducibility of decision making. The HT panel for the review process did not include members involved in the original discussion. Results During 2010, 108 meetings were held, attended by a median of 3 interventional cardiologists, 1 non-interventional cardiologist and 2 cardiac surgeons. A total of 1454 cases were discussed (mean 13.5 cases per meeting). 854 cases $(58.7 \%)$ were from our own unit and $600(41.3 \%)$ from referring hospitals. $356(24.5 \%)$ were current inpatients and 1098 (75.5\%) were outpatients. 1340 (92.2\%) were patients with stable coronary artery disease (CAD) or ACS. The HT recommendation was for coronary artery bypass grafting $(\mathrm{CABG}) \pm$ valve surgery in $429(32 \%)$ cases, percutaneous coronary intervention (PCI) in 303 (22.6\%), and optimised medical therapy (OMT) in $264(19.7 \%)$. In the remaining 344 cases $(25.7 \%)$ further investigation was advised before a HT decision was made; the most frequent recommendation was for a pressure wire study, in 151 cases (43.9\%). 117 randomly selected cases were analysed to determine whether the HT recommendation had been actioned. This occurred in $101(86.3 \%)$ cases. In the remaining 16 cases, deviation from the initial plan was due to the patient declining revascularisation (CABG 3, PCI 1), development of new co-morbidity (2) or revascularisation of different vessels (6). The reason for deviation was unclear in four cases. 50 cases were re-presented after 1 year with the original HT recommendation being the same in 38 cases $(76 \%)$. Different decisions in the remaining 12 (24\%) included seven cases $(14 \%)$ in which further investigation had initially been suggested, and re-vascularisation was recommended on re-presentation.

Conclusions A well-structured HT allows a large number of cases to be evaluated while interdisciplinary discussion facilitates consensus with evidence-based and individualised advice. There is a prominent role for pressure wire assessment in the further evaluation of equivocal CAD. The HT approach appears robust and reproducible in the majority of cases. Variation in decision making reflects the equipoise between suitability of CABG, PCI and OMT in many cases.

\section{NUCLEAR MAGNETIC RESONANCE PROFILING OF SERUM IDENTIFIES NOVEL BIOMARKER ASSOCIATED WITH CORONARY ATHEROSCLEROSIS}

doi:10.1136/heartjnl-2012-301877b.15

${ }^{1} \mathrm{~S}$ T Tan, ${ }^{1}{ }^{1} \mathrm{~J}$ Sehmi, ${ }^{1}$ A Al-Hussaini, ${ }^{2} \mathrm{M}$ Ala-Korpela, ${ }^{1}$ Paul Elliott, ${ }^{1} \mathrm{~J}$ C Chambers, ${ }^{1} \mathrm{~J} S \mathrm{~S}$ Kooner. ${ }^{1}$ Imperial College London, London, UK; ${ }^{2}$ University of Oulu, Oulu, Finland

Introduction Coronary heart disease (CHD) is the leading cause of death worldwide. Current CHD risk prediction tools do not accurately identify risk of $\mathrm{CHD}$ in all populations. There is an urgent need for discovery of novel biomarkers to help understand mechanisms underlying CHD and improve risk functions to identify people at increased risk. We used serum metabonomic nuclear magnetic resonance (NMR) profiling to identify biomarkers associated with the presence of coronary artery calcification (CAC), a quantitative measure of coronary atherosclerosis.

Methods We investigated 2358 men and women aged 35-75 years participating in the London Life Sciences Population (LOLIPOP) Study. Participants with prior history of CHD were excluded. All participants completed a structured health questionnaire, underwent physical assessment and had blood collected after an $8 \mathrm{~h}$ fast. CAC was quantified using electron beam CT. Quantitative NMR spectroscopy was used to characterise serum samples for lipoproteins, fatty acids and low molecular weight markers. Linear regression was used to determine the relationship between metabolites and Agatston score. Statistical significance was inferred at $p<0.0013$, corresponding to a Bonferroni correction for 39 primary NMR measures. Results CAC was associated with older age, male gender, cigarette smoking, raised systolic and diastolic blood pressure, obesity and hypercholesterolaemia. Among the 39 NMR measures, 11 were associated with CAC after adjustment for age, sex and ethnicity $\left(p=0.0013\right.$ to $\left.p=5.0 \times 10^{-12}\right)$. In multivariate analysis, after further adjustment for conventional risk factors, we identified an association of the metabonomic biomarker (an abundant naturally occurring nonessential amino acid) with coronary artery calcium $\left(p=7.9 \times 10^{-4}\right)$. The OR for the presence of significant coronary atherosclerosis (CAC>100) was 2.21 (95\% CI 1.59 to $3.07, p=2.2 \times 10^{-6}$ ) among people in the lowest quartile for the novel metabolite, compared to those in the highest quartile, implying that lower levels of the metabonomic biomarker confers increased CHD risk.

Conclusions Using NMR spectroscopy, we have identified a novel metabonomic biomarker to be independently associated with CAC, a measure of coronary atherosclerosis. Further testing in large prospective cohorts is required to evaluate the predictive value of this novel metabonomic biomarker to CHD. 